\title{
APLICAÇÃO DO DELINEAMENTO SIMPLEX-CENTROIDE NO ESTUDO DA CINÉTICA DA OXIDAÇÃO DE BIODIESEL B100 EM MISTURA COM ANTIOXIDANTES SINTÉTICOS
}

\author{
Dionísio Borsato*, Luiz Henrique Dall'Antonia, Carmen Luísa B. Guedes, Elaine Cristina R. Maia, Heverson Renan de \\ Freitas, Ivanira Moreira e Kelly Roberta Spacino \\ Departamento de Química, Universidade Estadual de Londrina, CP 6001, 86051-990 Londrina - PR, Brasil
}

Recebido em 11/1/10; aceito em 24/5/10; publicado na web em 24/8/10

\begin{abstract}
THE SIMPLEX-CENTROID DESIGN APPLIED TO STUDY OF THE KINETICS OF THE OXIDATION OF B100 BIODIESEL IN BLEND WITH SYNTHETIC ANTIOXIDANTS. Antioxidants are an alternative to prevent or slow the degradation of the biofuel. In this study, it was evaluated the oxidative stability of B100 biodiesel from soybean oil in the presence of three commercial synthetic antioxidants, butylated hydroxyanisole (BHA), butylated hydroxytoluene (BHT) and tert-butylhydroquinone (TBHQ), pure or blended, from the experimental design of simplex-centroid mixture. The reaction order and rate constant were also calculated for all tests. The treatment containing pure TBHQ proved to be the most effective, proven by design, the optimum mix obtained and the rate constant. Binary and ternary mixtures containing TBHQ also showed appreciable antioxidant effect.
\end{abstract}

Keywords: antioxidants, design of mixing and biofuel.

\section{INTRODUÇÃO}

Os combustíveis fósseis, usados atualmente em larga escala como fonte de energia são recursos finitos e extremamente poluidores. Diante da necessária busca por fontes de energias limpas e renováveis, o estudo de biocombustíveis tem se apresentado como uma alternativa viável para a solução destes problemas. ${ }^{1}$

Dentre os biocombustíveis destaca-se o biodiesel, que pode ser definido como sendo um mono-alquil éster de ácido graxo derivado de fontes renováveis, podendo ser produzido a partir da reação de óleos vegetais ou gordura animal com um álcool, geralmente metanol, na presença de um catalisador, normalmente uma base forte, como hidróxido de sódio ou de potássio. Este método de produção é conhecido como transesterificação. ${ }^{2-6}$

Algumas características devem ser consideradas para o biodiesel como ter boa biodegradabilidade, ter uma inerente lubrificidade na forma pura, apresentar competitividade com o diesel em termos de propriedades combustíveis e, além disso, não contribui para a emissão de aromáticos e óxidos de enxofre. ${ }^{7}$

No entanto, ao contrário dos combustíveis fósseis, que são relativamente inertes e mantêm as suas características essenciais pouco alteradas ao longo da estocagem, o biodiesel degrada mais rapidamente com o tempo e pode alterar-se devido à ação do ar, da luz, temperatura e umidade. O seu contato com contaminantes, tanto de natureza inorgânica quanto microbiana, também tende a introduzir variações na qualidade do produto, sendo a oxidação decorrente da sua exposição ao ar atmosférico um dos principais problemas de degradação a que o biodiesel está sujeito. ${ }^{6}$ Alterações de qualidade do biodiesel e misturas, como elevação da sua acidez, da sua corrosividade e a formação de produtos indesejáveis, como polímeros e depósitos, foram observadas ao longo do tempo de estocagem. ${ }^{8,9}$

A estabilidade à oxidação é, portanto, um parâmetro de grande importância para o controle da qualidade do biodiesel. O processo de degradação oxidativa do biodiesel depende da natureza dos ácidos graxos utilizados na sua produção associado, principalmente, ao grau de insaturação dos ésteres que o compõe, além da umidade, temperatura e absorção de luz. ${ }^{2,10}$

\footnotetext{
*e-mail: dborsato@uel.br
}

Assim, a instabilidade oxidativa do biodiesel é atribuída principalmente ao fato deste ter um alto teor de ésteres insaturados, os quais podem ser facilmente oxidados, como o linoleato de metila $\left(\mathrm{C}_{18: 2}\right)$ e o linolenato de metila $\left(\mathrm{C}_{18: 3}\right)$, levando à formação de compostos de decomposição como ácidos, aldeídos, ésteres, cetonas, peróxidos e alcoóis. ${ }^{11}$ Estes produtos não afetam apenas as propriedades do biodiesel, mas também trazem problemas de funcionamento ao motor. ${ }^{12}$ Esta instabilidade é uma grande barreira ao aumento da aceitação do biodiesel por fabricantes de motores e, consequentemente, ao aumento do mercado para esse combustível. ${ }^{13}$

As cadeias de ácidos graxos têm variados níveis de insaturação, com duplas ligações em configuração cis. Quando múltiplas insaturações estão presentes, elas não se encontram conjugadas e sim separadas por um grupo metileno. Tais grupos adjacentes a duas insaturações são sítios de oxidação extremamente reativos por sofrerem uma imediata isomerização dando origem a estruturas conjugadas estáveis, as quais reagem diretamente com o oxigênio, formando um peróxido. ${ }^{14}$

Para o óleo de soja, uma importante matéria-prima para o biodiesel no Brasil, cerca de $76 \%$ de seus triglicerídeos são formados por ácidos graxos insaturados, dos quais $51 \%$ correspondem ao ácido linoleico $\left(\mathrm{C}_{18: 2}\right){ }^{15}$ Esse caráter insaturado acaba incorporado pelo biocombustível. Dessa forma, a estabilidade oxidativa vem sendo bastante investigada pela comunidade científica, tanto para o biodiesel quanto para algumas oleaginosas. ${ }^{16}$

A rancificação oxidativa não ocorre normalmente com ácidos graxos saturados, já que a formação de radicais livres, neste caso, é energeticamente desfavorável. A oxidação é proveniente de sucessivas reações radicalares que ocorrem nas insaturações dos ésteres das cadeias graxas, em contato com o oxigênio atmosférico, sendo acelerada pela presença de íons metálicos, luz, temperatura, radiação ionizante e outros agentes oxidantes, de modo a formar peróxidos e, consequentemente, outros produtos oxidativos. ${ }^{8-10}$

Com a finalidade de inibir ou retardar a oxidação lipídica de óleos, gorduras e alimentos gordurosos são empregados compostos químicos conhecidos como antioxidantes e/ou estabilizadores. ${ }^{17}$

Os antioxidantes ocorrem naturalmente em óleos vegetais e os mais comuns são os tocoferóis. No entanto, alguns processos de produção de óleos vegetais incluem uma etapa de destilação para sua purificação. O biodiesel obtido a partir destes óleos normalmente 
possui pouco ou nada de antioxidantes naturais, assim, torna-se menos estável. ${ }^{14}$ Surge então a necessidade de aplicar antioxidantes sintéticos para aumentar a estabilidade do biocombustível, e assim manter suas propriedades por um período maior.

$\mathrm{O}$ uso de antioxidantes e seus mecanismos funcionais tem sido amplamente estudado e podem ser classificados em primários, sinergistas, removedores de oxigênio, biológicos, agentes quelantes e antioxidantes mistos. Os antioxidantes primários promovem a remoção ou inativação dos radicais livres formados durante a iniciação ou propagação da reação, através da doação de átomos de hidrogênio a estas moléculas, interrompendo a reação em cadeia. ${ }^{18}$ Os principais antioxidantes e mais conhecidos deste grupo são os compostos fenólicos sintéticos, tais como butil-hidroxianisol (BHA), butil-hidroxitolueno (BHT), terc-butil-hidroquinona (TBHQ) e propil galato (PG), e naturais como os tocoferóis. ${ }^{18}$

$\mathrm{O}$ desenvolvimento de qualquer produto envolvendo mais de um ingrediente requer algumas formas particulares de experimentos para a mistura ${ }^{19}$. Delineamentos para mistura são empregados em vários experimentos para o desenvolvimento de produtos. As respostas dependem somente das proporções dos componentes presentes na mesma e não da quantidade absoluta na mistura. ${ }^{20}$

Uma dificuldade encontrada em muitos experimentos para mistura é que alguns dos componentes estão sujeitos a limites superior e/ou inferior. Tais limites podem produzir regiões do delineamento com formas diferenciadas para a qual é impossível utilizar alguns delineamentos. Foi proposta uma solução para este problema através da realização dos experimentos em pontos extremos e vários centroides na região de restrição do delineamento. ${ }^{21}$ Esses procedimentos são conhecidos como delineamentos de vértices extremos e, como os delineamentos de Scheffé, permitem explorar toda a região experimental..$^{22}$

Portanto, a formulação ótima de um produto não é estritamente um problema tecnológico, tão pouco um problema comercial, somente. Em geral, ambas as áreas oferecem restrições que contribuem para determinar quais formulações são possíveis. ${ }^{23}$

Para fazer um bom uso dos métodos de otimização é necessário que os resultados sejam utilizados como um ponto de partida para a tomada de decisão. Os métodos de otimização têm sido aplicados de maneira variada na indústria de processos químicos, térmicos e gerenciamento para redução de custos. ${ }^{24-26}$

Em relação à obtenção de formulações ótimas, os delineamentos de mistura podem gerar várias respostas simultaneamente, o que pode dificultar a interpretação da melhor solução. ${ }^{24,25}$ Porém, Derringer e Suich ${ }^{26}$ sugeriram um método para determinar a melhor combinação de múltiplas respostas com restrições.

O objetivo do presente trabalho foi apresentar uma análise da eficiência de antioxidantes sintéticos, utilizando delineamento experimental de misturas, quando adicionados ao biodiesel B100, bem como estudar a cinética de oxidação do biodiesel através do acompanhamento da reação de oxidação, na temperatura de $50{ }^{\circ} \mathrm{C}$, na presença de antioxidantes.

\section{PARTE EXPERIMENTAL}

\section{Biodiesel}

As amostras de biodiesel foram obtidas a partir da reação de transesterificação de óleo de soja por metanol e cedidas pelo Departamento de Química da UNICENTRO-PR.

\section{Análise cromatográfica}

Foi utilizado um cromatógrafo modelo GC-17A com detector de ionização de chama Shimadzu, com uma coluna DB1 (J\&W Scienti- fic) $-100 \%$ polimetilsiloxano com $30 \mathrm{~m}$ de comprimento x $0,25 \mathrm{~mm}$ de diâmetro interno x $0,25 \mu \mathrm{m}$ de espessura de filme. A temperatura do injetor split foi de $340^{\circ} \mathrm{C}$ e a temperatura do detector de $340{ }^{\circ} \mathrm{C}$. A rampa de aquecimento da coluna foi mantida inicialmente à temperatura de $50{ }^{\circ} \mathrm{C}$ por 2 min e, após isso, aquecida na razão de $10^{\circ} \mathrm{C} /$ min até $180^{\circ} \mathrm{C}$ permanecendo nessa temperatura por $2 \mathrm{~min}$; a seguir, foi aquecida à razão de $15^{\circ} \mathrm{C} / \mathrm{min}$ até $340^{\circ} \mathrm{C}$, permanecendo nessa temperatura por $10 \mathrm{~min}$. A vazão do gás de arraste, $\mathrm{N}_{2}$, foi de $1,5 \mathrm{~mL} /$ min e o volume de injeção de $2,0 \mu \mathrm{L}$ com razão de split 10 . Os dados foram coletados por meio do software CLASS-CR10 Shimadzu.

\section{Antioxidantes}

Foram utilizados os antioxidantes grau P. A. butil-hidroxianisol (BHA) Synth, butil-hidroxitolueno (BHT) Synth e Terc-Butil-hidroquinona (TBHQ) Acros, cujas quantidades, não ultrapassando 6,0 × 10-3 $\mathrm{mol} / \mathrm{L}$, foram estabelecidas pelo delineamento de mistura e adicionados diretamente ao biodiesel antes da avaliação da estabilidade oxidativa.

\section{Avaliação da estabilidade oxidativa pelo teste de oxidação em estufa}

As amostras de biodiesel contendo as quantidades de antioxidantes estabelecidas pelo delineamento simplex-centroide, bem como as amostras controle, foram levadas ao aquecimento em estufa a 50 ${ }^{\circ} \mathrm{C}$ durante tempo necessário para que todas as amostras atingissem o índice de peróxido máximo. As amostras do controle e do ponto central foram em triplicata e nos demais pontos em amostra única, sendo o índice de peróxido determinado ao término de cada semana. ${ }^{10}$ A análise foi repetida uma vez para os pontos com amostragem única.

\section{Índice de peróxido (I. P.)}

Foi determinado por titulação com tiossulfato de sódio pelo método AOAC Cd 8-53. ${ }^{27}$

\section{Delineamento experimental para mistura ${ }^{28}$}

Foi utilizado o planejamento experimental de mistura simplexcentroide (Figura 1) para avaliar o efeito da adição dos antioxidantes BHA $\left(\mathrm{x}_{1}\right)$, BHT $\left(\mathrm{x}_{2}\right)$ e TBHQ $\left(\mathrm{x}_{3}\right)$ em biodiesel B100 obtido do óleo de soja. O delineamento para três componentes foi composto de 7 ensaios com duas repetições no ponto central. ${ }^{29}$

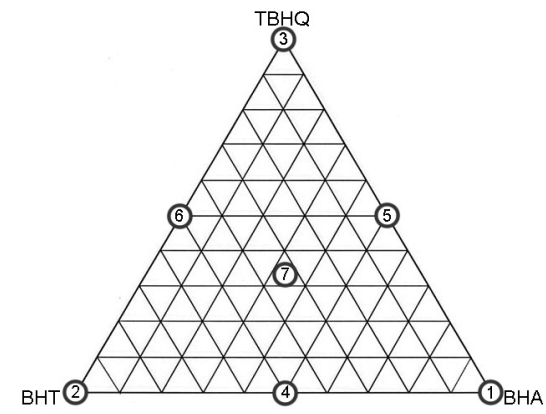

Figura 1. Delineamento experimental simplex centroide para 3 componentes

Modelo matemático

A função utilizada foi do tipo

$$
y=\sum_{1 \leq i \leq q} \gamma_{i}^{\circ} x_{i}+\sum_{1 \leq i \leq j \leq q} \gamma_{i j}^{\circ} x_{i} x_{j}+\gamma_{123}^{\circ} x_{1} x_{2} x_{3}
$$


onde y representa a função resposta dos dados experimentais; $\mathrm{x}_{1}, \mathrm{x}_{2} \mathrm{e}$ $\mathrm{x}_{3}$ são as variáveis independentes e correspondem à porcentagem de BHA, BHT e TBHQ na mistura, respectivamente, e $\gamma$ os parâmetros estimados. ${ }^{20}$

\section{Análise estatística}

O teste de Tukey aplicado às médias e os coeficientes de regressão foram estimados utilizando o software Statistica v.9.0.22

\section{RESULTADOS E DISCUSSÃO}

A análise cromatográfica mostrou que o biodiesel utilizado era formado basicamente pelos ésteres palmitato de metila, oleato de metila, linoleato de metila, linolenato de metila bem como outros ésteres metílicos cuja composição varia do C8:0 até C22:0. Os ésteres com composição química variando de $\mathrm{C} 8$ a C14, respectivamente, representaram $0,50 \%$ da amostra do biodiesel utilizado. O teor de éster metílico do ácido graxo C16:0 foi de 19,80\% e dos ésteres metílicos C18:0; C18:1 e C18:2 foi de $73,80 \%$. Os ésteres formados por C20 a C22 representaram 3,57\% da amostra de biodiesel utilizado. Portanto, estes ésteres juntos somam 97,67\% das substâncias presentes no biodiesel B100 utilizado, estando em acordo com a especificação da União Europeia, onde os teores de ácidos graxos livres, álcool, glicerina e água devem ser mínimos, de modo que a pureza do biodiesel deve ser maior que $96,5 \% .{ }^{30}$ Além disso, os teores de mono, di e triglicerídeos presentes somaram juntos $0,37 \%$ e o teor de glicerina livre verificado foi de $2,0 \mathrm{mg} / 100 \mathrm{~g}$ de amostra, valor bastante inferior ao da legislação brasileira, que prevê um teor máximo de glicerina livre de $0,02 \%(\mathrm{~m} / \mathrm{m}) .{ }^{31}$

As respostas expressas em valores médios dos 7 ensaios e do controle, para o índice de peróxido do biodiesel, utilizando o teste de estabilidade acelerada em estufa, estão apresentadas na Tabela 1, de acordo com o tempo de ensaio. Os resultados do delineamento experimental mostraram que, em todas as proporções, os antioxidantes retardaram a oxidação do biodiesel quando comparada com o controle, com exceção do tratamento 1 para o $7^{\circ}$ dia de ensaio que apresentou valor de I. P. maior que o do controle. Porém, o teste de Tukey aplicado a estes dois tratamentos não apresentou diferença significativa em nível de 5\%. Pode-se observar também que o tratamento 3, no qual foi empregado TBHQ como único antioxidante, apresentou o menor índice de peróxido em todos os ensaios, fato também observado por Domingos et al. quando estudaram a estabilidade oxidativa do biodiesel de óleo de soja. ${ }^{32}$ Observou-se também que o BHA apresentou menor eficiência quando comparado com o BHT e TBHQ.

Tabela 1. Valores médios dos índices de peróxido obtidos segundo o delineamento experimental de mistura simplex-centroide

\begin{tabular}{lccccc}
\hline \multirow{2}{*}{ Tratamento } & \multirow{2}{*}{ Mistura* } & \multicolumn{4}{c}{ Índice de peróxido (meq/ kg de amostra) } \\
& & $7^{\circ}$ dia & $14^{\circ}$ dia & $21^{\circ}$ dia & $24^{\circ}$ dia \\
\hline 1 & $(1 ; 0 ; 0)$ & 117,77 & 149,06 & 236,13 & 307,09 \\
2 & $(0 ; 1 ; 0)$ & 78,72 & 88,66 & 118,84 & 128,60 \\
3 & $(0 ; 0 ; 1)$ & 22,44 & 28,64 & 41,00 & 55,16 \\
4 & $(1 / 2 ; 1 / 2 ; 0)$ & 105,94 & 149,22 & 257,69 & 491,74 \\
5 & $(1 / 2 ; 0 ; 1 / 2)$ & 32,53 & 57,74 & 84,85 & 136,64 \\
6 & $(0 ; 1 / 2 ; 1 / 2)$ & 32,15 & 48,06 & 67,14 & 115,16 \\
7 & $(1 / 3 ; 1 / 3 ; 1 / 3)$ & 66,92 & 75,47 & 99,35 & 149,11 \\
Controle & $(0 ; 0 ; 0)$ & 114,45 & 405,87 & 944,42 & 1019,81 \\
\hline
\end{tabular}

$*\left(\%_{\mathrm{BHA}}, \%_{\mathrm{BHT}}, \%_{\text {TBнQ }}\right)$
De acordo com Frankel, os métodos de estabilidade acelerada apresentam validade restrita, pois, à medida que se submete a amostra ao aquecimento, à luz ou ao contato com metais, muda-se o mecanismo da reação de oxidação, sendo que os testes efetuados em temperatura ambiente aproximam-se da estocagem real. Porém, por proporcionarem resultados mais rápidos, os métodos acelerados reduzem o tempo de trabalho e o consumo de reagentes e, segundo Frankel, o método de estufa é o que apresenta menores limitações quando comparado com outros métodos. ${ }^{33}$

Com a aplicação do delineamento experimental de mistura simplexcentroide, os modelos cúbicos a seguir, representados pelas Equações $2,3,4$ e 5 , foram obtidos para o $7^{\circ}, 14^{\circ}, 21^{\circ}$ e $24^{\circ}$ dias de ensaio, respectivamente, e os coeficientes de determinação ajustados para as equações foram de 91,$83 ; 98,46 ; 93,95$ e $92,70 \%$, respectivamente. ${ }^{28}$

$$
\begin{aligned}
& y_{7}=117,8 x_{1}+78,7 x_{2}+22,4 x_{3}+30,8 x_{1} x_{2}-150,3 x_{1} x_{3} \\
& -73,7 x_{2} x_{3}+416,3 x_{1} x_{2} x_{3} \\
& y_{14}=149,1 x_{1}+88,7 x_{2}+28,6 x_{3}+121,5 x_{1} x_{2}-124,4 x_{1} x_{3} \\
& -42,4 x_{2} x_{3}-223,7 x_{1} x_{2} x_{3}
\end{aligned}
$$

$y_{21}=236,1 x_{1}+118,8 x_{2}+41,0 x_{3}+320,8 x_{1} x_{2}-214,9 x_{1} x_{3}$

$-51,1 x_{2} x_{3}-1045,8 x_{1} x_{2} x_{3}$

$y_{24}=307,1 x_{1}+128,6 x_{2}+55,2 x_{3}+1095,6 x_{1} x_{2}-177,9 x_{1} x_{3}$

$+93,1 x_{2} x_{3}-3424,0 x_{1} x_{2} x_{3}$

Os altos coeficientes e a análise de variância mostraram que tais equações podem ser utilizadas para fins preditivos. As mesmas equações mostram que o efeito da ação do TBHQ, isoladamente ou em combinação binária ou ternária com os outros dois antioxidantes, é muito eficaz no retardamento da reação de oxidação, com exceção da mistura ternária para o $7^{\circ}$ dia onde o coeficiente apresentou valor positivo (Equação 2) diferentemente das outras equações.

A região de combinação entre as três variáveis $\mathrm{x}_{1}, \mathrm{x}_{2}$ e $\mathrm{x}_{3}$ pode ser observada através das curvas de nível, obtidas pelos modelos matemáticos, apresentadas nas Figuras 2, 3, 4 e 5, correspondentes ao $7^{\circ}, 14^{\circ}, 21^{\circ}$ e $24^{\circ}$ dias, respectivamente. Todas mostram que concentrações ao redor de $100 \%$ de TBHQ, regiões mais claras, são mais eficientes na proteção do biocombustível estudado, pois forneceram os menores índices de peróxidos no período analisado.

A otimização, utilizando o software Statistica, indicou a formulação contendo $100 \%$ de TBHQ como a melhor condição para evitar o processo oxidativo do biodiesel B100 obtido de óleo de soja para todos os períodos, até o $24^{\circ}$ dia de ensaio. O valor ótimo obtido coincide com o valor utilizado no tratamento 3 , conforme Tabela 1 .

A fim de comprovar a eficiência dos antioxidantes no retardamento da auto-oxidação do biodiesel B100, aplicou-se o teste Tukey ${ }^{28}$ comparando-se os valores obtidos das amostras contendo antioxidante e o controle. A partir deste teste, pode-se observar os tratamentos que apresentaram diferenças significativas com o controle e, assim, comprovar se o efeito antioxidante foi efetivo. Para o $7^{\circ}$ dia os tratamentos 3, 5 e 6, contendo TBHQ isoladamente ou em mistura binária com outro antioxidante, apresentaram diferenças significativas em relação ao controle. Para o $14^{\circ}, 21^{\circ}$ e $24^{\circ}$ dia todos os tratamentos apresentaram valores consideravelmente menores que os obtidos para o controle, comprovando, portanto, a eficiência dos mesmos.

Para cálculos estatísticos, consideraram-se apenas os primeiros 24 dias de ensaio, pois este foi o tempo necessário para que o controle 


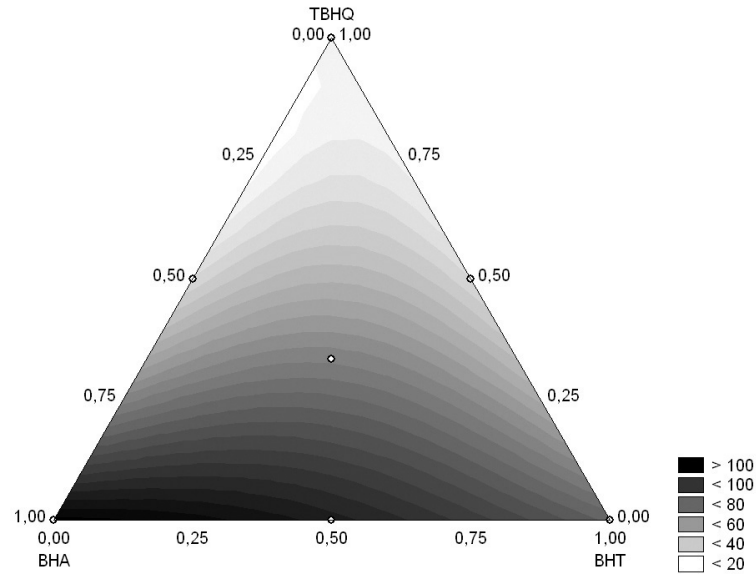

Figura 2. Região de combinação entre as três variáveis $x_{1}, x_{2}$ e $x_{3}$ obtida por meio da Equação 2 para os índices de peróxido correspondentes ao $7^{\circ}$ dia de ensaio

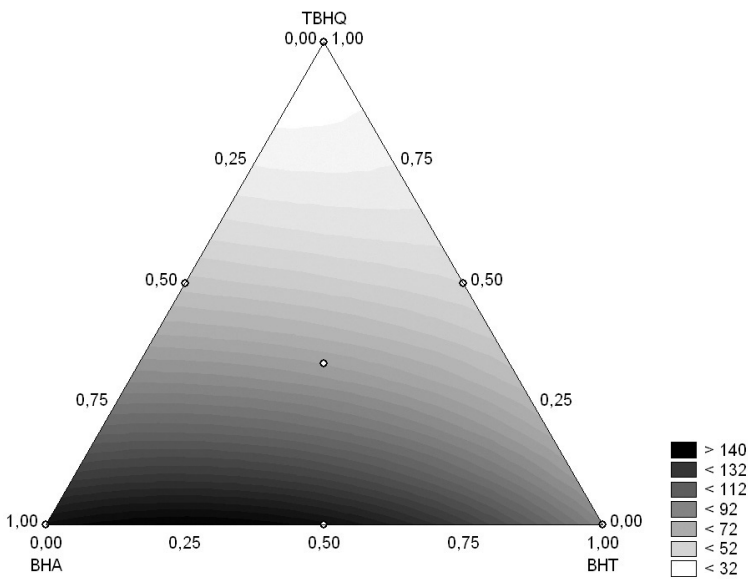

Figura 3. Região de combinação entre as três variáveis $x_{p}, x_{2}$ e $x_{3}$ obtida por meio da Equação 3 para os índices de peróxido correspondentes ao $14^{\circ}$ dia de ensaio

atingisse o I. P. máximo, começando então a diminuir. As análises, porém, continuaram até que todas as amostras apresentassem o mesmo comportamento. A Figura 6 mostra as curvas ajustadas para os índices de peróxido em função do tempo para todas as amostras. O I. P. inicial do biodiesel utilizado foi de $5,14 \mathrm{meq} / \mathrm{kg}$ de amostra.

Apesar do índice de peróxido não ser o mais indicado para o monitoramento da oxidação, devido à formação de produtos secundários, ${ }^{34}$ a Figura 6, bem como os estudos feitos por Ferrari e Souza, ${ }^{10}$ mostraram que as curvas obtidas não apresentaram oscilações significativas.

Na Figura 6 pode-se observar que a iniciação, que corresponde ao período de indução que precede a oxidação, foi retardada em todos os tratamentos contendo os antioxidantes sintéticos. O período de indução do processo de oxidação dos ésteres metílicos, nos primeiros 24 dias de estudo, mostrou que o biodiesel contendo apenas TBHQ apresentou o menor valor do I. P., seguido da mistura binária contendo BHT com TBHQ. Ao contrário, a mistura de BHA com BHT e BHA isoladamente apresentaram, neste intervalo, menor período de indução.

A Figura 6 mostra ainda a propagação, uma fase exponencial, onde os radicais livres formados reagem com o substrato para dar origem a novos radicais livres e hidroperóxidos. ${ }^{33,34}$ Para a amostra controle, esse período inicia na primeira semana e, para o tratamento com TBHQ e aquele com a mistura binária contendo BHT e TBHQ, ele inicia após 35 dias de ensaio. Muitos pesquisadores têm indicado

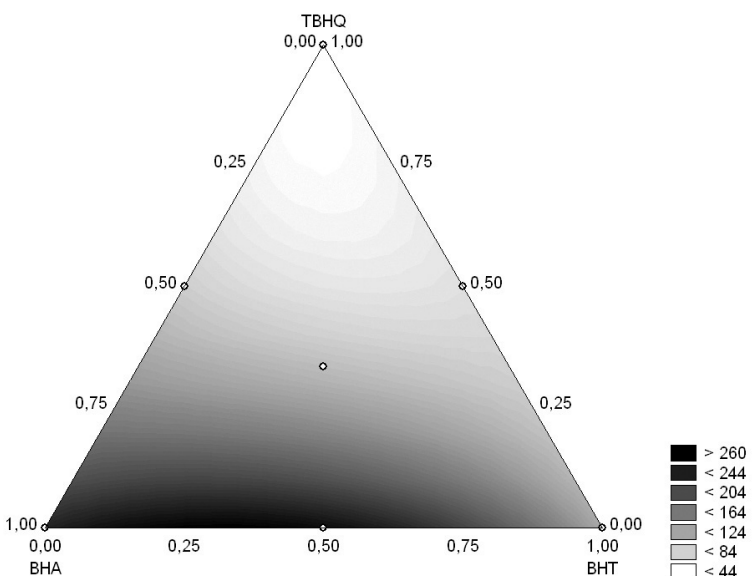

Figura 4. Região de combinação entre as três variáveis $x_{1}, x_{2}$ e $x_{3}$ obtida por meio da Equação 4 para os índices de peróxido correspondentes ao $21^{\circ}$ dia de ensaio

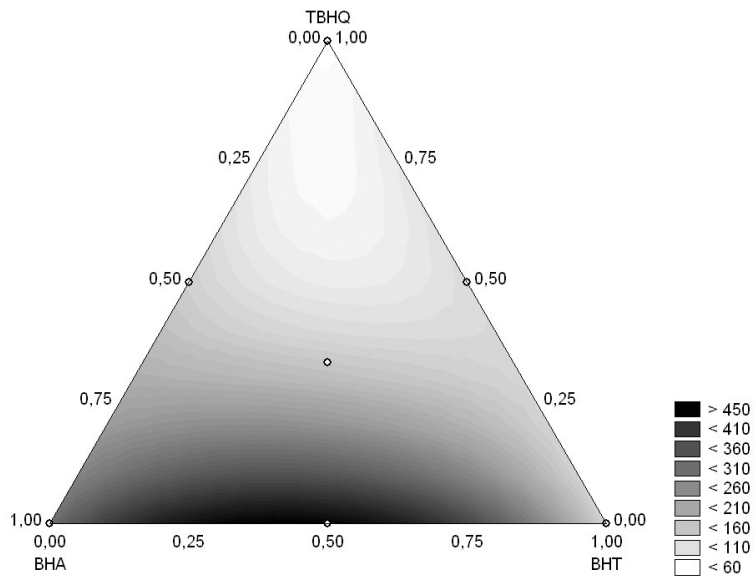

Figura 5. Região de combinação entre as três variáveis $x_{1}, x_{2}$ e $x_{3}$ obtida por meio da Equação 5 para os índices de peróxido correspondentes ao $24^{\circ}$ dia de ensaio

que TBHQ é muito mais eficaz do que os outros aditivos porque inibe tanto a oxidação primária como a secundária. ${ }^{34}$ Por fim, temse a terminação que corresponde à formação de produtos estáveis, produtos secundários de oxidação, obtidos por cisão, e rearranjos dos peróxidos como epóxidos, compostos voláteis e não voláteis. ${ }^{14,34,35}$

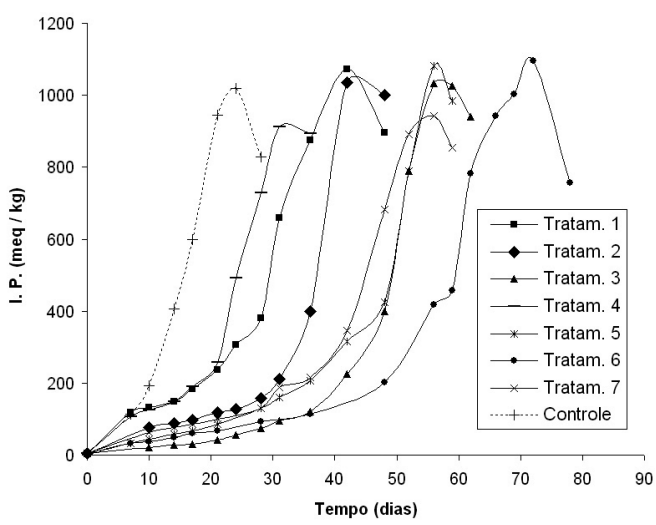

Figura 6. Curvas ajustadas e dados experimentais para o índice de peróxido em função do tempo para os 7 tratamentos e o controle 
A Tabela 2 mostra o período em que foi observado o maior índice de peróxido para cada uma das amostras, incluindo o controle. Os tratamentos 3, 5, 6 e 7, os quais continham TBHQ, foram os que apresentaram maior tempo para atingir o índice de peróxido máximo, com destaque para a mistura binária BHT e TBHQ (tratamento 6) que apresentou atividade antioxidante por 16 dias a mais.

Tabela 2. Tempo, em dias, para que o índice de peróxido máximo fosse atingido para os 7 tratamentos e o controle

\begin{tabular}{ccc}
\hline Tratamento & Mistura* & Tempo (dia) \\
\hline 1 & $(1 ; 0 ; 0)$ & 42 \\
2 & $(0 ; 1 ; 0)$ & 42 \\
3 & $(0 ; 0 ; 1)$ & 56 \\
4 & $(1 / 2 ; 1 / 2 ; 0)$ & 31 \\
5 & $(1 / 2 ; 0 ; 1 / 2)$ & 56 \\
6 & $(0 ; 1 / 2 ; 1 / 2)$ & 72 \\
7 & $(1 / 3 ; 1 / 3 ; 1 / 3)$ & 56 \\
Controle & $(0 ; 0 ; 0)$ & 24 \\
\hline
\end{tabular}

$*\left(\%_{\text {BHA }}, \%_{\text {BHT }}, \%_{\text {TBHQ }}\right)$

Quando não se tem a possibilidade de conhecer o verdadeiro mecanismo da reação pode-se propor uma equação de ordem n mais geral para o ajustamento dos dados. ${ }^{36}$ De acordo com Petriella et al., quando a curva da reação em função do tempo apresenta um período de indução mais longo seguido de um rápido crescimento recomendase aplicar uma ordem de reação $n$ variando de 0 a 1 indicando uma reação mais complexa. ${ }^{36}$ Desse modo, a ordem da reação foi obtida utilizando-se a Equação 6:

$$
v=\frac{d C}{d t}=k C^{n}
$$

onde $n$ é a ordem $(0 \leq n<1), C$ o produto da reação e $k$ a constante de velocidade. ${ }^{36}$

Outro importante parâmetro obtido, também utilizado para avaliar a eficiência de cada tratamento, foi a constante de velocidade $k$ para oxidação do biodiesel. Integrando-se e logaritmando a Equação 6, obteve-se a Equação 7, que correlaciona linearmente o logaritmo do tempo com o logaritmo do índice de peróxido.

$$
\log C=\left(\frac{1}{1-n}\right) \log [(1-n) k]+\left(\frac{1}{1-n}\right) \log t
$$

Desta forma, a partir dos coeficientes angulares e lineares obtidos para cada uma das amostras, pode-se calcular primeiramente a ordem da reação $n$ e, após, a constante de velocidade. O índice de peróxido inicial foi subtraído de cada um dos I. P. obtidos antes dos cálculos. A Figura 7 mostra as retas ajustadas pelo método dos mínimos quadrados para os 7 tratamentos e o controle.

Os valores de $n$ e $k$ obtidos e o coeficiente de correlação linear $r$ de cada reta encontram-se na Tabela 3. Verifica-se que a ordem de reação, n, ficou entre 0 e 1 indicando uma reação mais complexa e que o modelo cinético postulado (Equação 6) foi adequado.

Os coeficientes de correlação obtidos mostram que as equações de retas obtidas apresentaram um ajuste razoável, indicando que elas podem ser usadas para fins preditivos. As ordens das reações e as constantes de velocidade obtidas para os tratamentos 5, 6 e 7 mostraram certa similaridade de comportamento entre as misturas contendo TBHQ.

Como esperado, os menores valores de $k$ foram obtidos para os ensaios contendo TBHQ, puro ou em mistura binária ou ternária, com

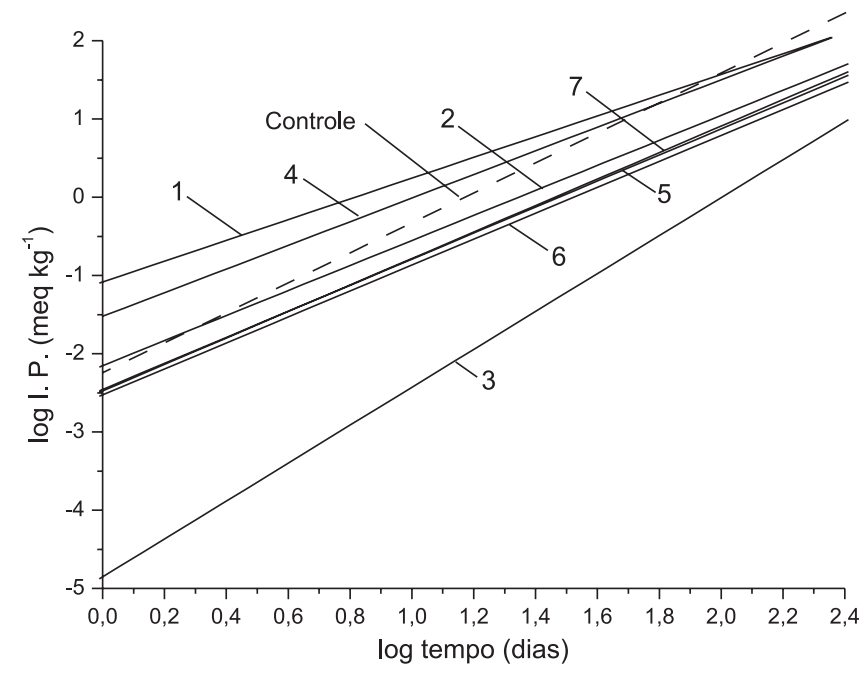

Figura 7. Retas ajustadas do logaritmo de tempo em função do logaritmo do índice de peróxido para os 7 tratamentos e o controle

Tabela 3. Coeficiente de correlação linear $r$, ordem da reação $n$ e a constante de velocidade $k$ para os 7 tratamentos e o controle

\begin{tabular}{ccccc}
\hline Tratamento & Mistura* $^{*}$ & $\mathrm{r}$ & $\mathrm{n}$ & $\mathrm{k}$ \\
\hline 1 & $(1 ; 0 ; 0)$ & 0,93507 & 0,24592 & 0,202199 \\
2 & $(0 ; 1 ; 0)$ & 0,86861 & 0,37475 & 0,072112 \\
3 & $(0 ; 0 ; 1)$ & 0,95253 & 0,58737 & 0,024087 \\
4 & $(1 / 2 ; 1 / 2 ; 0)$ & 0,92904 & 0,33837 & 0,148503 \\
5 & $(1 / 2 ; 0 ; 1 / 2)$ & 0,95546 & 0,39940 & 0,055654 \\
6 & $(0 ; 1 / 2 ; 1 / 2)$ & 0,94953 & 0,39673 & 0,049624 \\
7 & $(1 / 3 ; 1 / 3 ; 1 / 3)$ & 0,94018 & 0,40907 & 0,058245 \\
Controle & $(0 ; 0 ; 0)$ & 0,99625 & 0,47807 & 0,129103 \\
\hline$\left.\%_{\text {BнА }}, \%_{\text {ВНТ }} \%_{\text {твнQ }}\right)$ & & &
\end{tabular}

destaque para o tratamento 3, que apresentou a menor de todas as constantes de velocidade, caracterizando o excelente efeito antioxidante do terc-butil-hidroquinona sobre o biodiesel B100 de óleo de soja.

\section{CONCLUSÃO}

Através dos resultados obtidos verificou-se que o tratamento contendo o antioxidante TBHQ, isoladamente, mostrou-se o mais eficiente para aumentar a estabilidade oxidativa de biodiesel B100 de óleo de soja, fato comprovado pelo delineamento, pela mistura ótima obtida e pela constante de velocidade. Misturas binárias e ternárias contendo TBHQ também apresentaram apreciável efeito antioxidante sobre o biocombustível.

\section{AGRADECIMENTOS}

À Fundação Araucária pelo suporte financeiro, à UEL e ao CNPq pela concessão de bolsas.

\section{REFERÊNCIAS}

1. Tashtoush, G. M.; Al-Widyan, M. I.; Al-Jarrh, M. M.; Energy Convers. Manage. 2004, 45, 696.

2. Ferrari, R. A.; Oliveira, V. S., Scabio, A.; Quim. Nova 2005, $28,19$.

3. Oliveira, F. C. C.; Suarez, P. A. Z.; Santos, W. L. P.; Quim. Nova na Escola 2008, n 8,3 . 
4. Gerpen, J. V.; Fuel Process Technol. 2005, 86, 1097.

5. Knothe, G.; Fuel Process Technol. 2005, 86, 1059.

6. Meher, L. C.; Sagar, D. V.; Naik, S. N.; Renewable Sustainable Energy Rev. 2006, 10, 248.

7. Knothe, G.; Fuel Process Technol. 2007, 88, 669.

8. Bondioli, P.; Gasparoli, A.; Della Bella, L.; Tagliabue, S.; Lacoste, F.; Lagardere, L.; Eur. J. Lipid Sci. Technol. 2003, 105, 735.

9. Bondioli, P.; Gasparoli, A.; Della Bella, L.; Tagliabue, S.; Lagardere, L.; Eur. J. Lipid Sci. Technol. 2004, 106, 822.

10. Ferrari, R. A.; Souza, W. L.; Quim. Nova 2009, 32, 106.

11. Xin, J.; Imahara, H.; Saka, S.; Fuel 2009, 88, 282.

12. Monyem, A.; van Gerpen, J. H.; Biomass Bioenergy 2001, $20,317$.

13. Dinkov, R.; Hristov, G.; Stratiev, D.; Aldayri, V. B.; Fuel 2008, 88, 732.

14. McCormick, R. L.; Ratcliff, M. M.; Lawrence, R.; Fuel Process Technol. 2007, 88, 651 .

15. Borsato, D.; Moreira, I.; Galão, O. F.; Detergentes Naturais e Sintéticos - Um Guia Técnico, 2a ed., Eduel: Londrina, 2004.

16. Ma, F.; Hanna, M. A.; Bioresour. Technol. 1999, 70, 1.

17. Alves, M. V.; Rocha, M. I.; Zotin, F. M. Z.; Pinto, R. R. C.; Bol. Téc. Petrobras 2006, 49, 65.

18. Ramalho, V. C.; Jorge, N.; Quim. Nova 2006, 29, 755.

19. Thompson, D. R.; Am. Soc. Agric. Engineers 1981, 24, 1077.

20. Cornell, J. A.; Experiments with mixtures - designs, models and the analysis of mixture data, $2^{\text {nd }}$ ed., John Wiley and Sons: New York, 1990.

21. McLean, R. A.; Anderson, V. L.; Technometrics 1966, 8, 447.
22. Steinberg, M. D.; Hunter, W. G.; Technometrics 1984, $26,71$.

23. Gacula, M. C.; Design and analysis of sensory optimization, Food \& Nutrition Press: Trumbull, 1993.

24. Chen, J. S.; Lee, C. M.; Crapo, C.; J. Food Sci. 1993, 58, 535.

25. Arteaga, G. E.; Li-Chan, E.; Vazquez-Arteaga, M. C.; Nakai, S.; Trends Food Sci. Technol. 1994, 5, 243.

26. Derringer, G.; Suich, R.; J. Qual. Technol. 1980, 12, 214.

27. A.O.C.S.; American Oil Chemist's Society Official Methods and recommended praticces of the American Oil Chemists Society, $5^{\text {th }}$ ed., A.O.C.S.: Champaign, 1998.

28. Statistica for Windows Software. v. 9.0, Tulsa, OK, USA, 2009.

29. Calado, V.; Montgomery, D. C.; Planejamento de Experimentos usando o Statistica, E-papers Serviços Editoriais Ltda: Rio de Janeiro, 2003.

30. Karaosmanoglu, F.; Becker, U. G.; Energy Sources 1996, 18, 637.

31. Brasil; Resolução no 7, de 19/3/ 2008 - ANP, Agência Nacional do Petróleo, Gás Natural e Biocombustíveis. Estabelece a especificação para a comercialização de biodiesel que poderá ser adicionado ao óleo diesel. Diário Oficial da União de 20/3/2008.

32. Domingos, A. K.; Saad, E. B.; Vechiatto, W.; Wilheim, H. M.; Ramos, L. P.; J. Braz. Chem. Soc. 2007, 18, 416.

33. Frenkel, E. N.; J. Sci. Food Agricult. 1991, 54, 495.

34. Jain, S.; Sharma, M. P.; Renewable Sustainable Energy Rev. 2010, 14, 667

35. Lillard, D. A.; Day, E. A.; J. Am. Oil Chem. Soc. 1964, 41, 549.

36. Petriella, C.; Resnik, S. L.; Lozano, R. D.; Chirife, J.; J. Food Sci. 1985, 50,622 . 